Lip

\title{
Return of the hardware floating-point elementary function
}

Jérémie Detrey, Florent de Dinechin,

Xavier Pujol

November 2006

École Normale Supérieure de Lyon

46 Allée d'Italie, 69364 Lyon Cedex 07, France

Téléphone : $+33(0) 4.72 .72 .80 .37$

Télécopieur : $+33(0) 4.72 .72 .80 .80$

Adresse électronique : lip@ens-lyon.fr

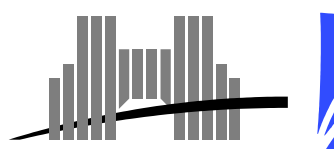




\title{
Return of the hardware floating-point elementary function
}

\author{
Jérémie Detrey, Florent de Dinechin, Xavier Pujol
}

November 2006

\begin{abstract}
The study of specific hardware circuits for the evaluation of floating-point elementary functions was once an an active research area, until it was realized that these functions were not frequent enough to justify dedicating silicon to them. Research then turned to software functions. This situation may be about to change again with the advent of reconfigurable co-processors based on field-programmable gate arrays. Such co-processors now have a capacity that allows to accomodate double-precision floating-point computing. Hardware operators for elementary functions targeted to such platforms have the potential to vastly outperform software functions, and will not permanently waste silicon resources. This article studies the optimization, for this target technology, of operators for the exponential and logarithm functions up to double-precision.
\end{abstract}

Keywords: Floating-point elementary functions, hardware operator, FPGA, exponential, logarithm.

\section{Résumé}

L'implantation en matériel dans les processeurs des fonctions élémentaires en virgule flottante a été un sujet de recherche actif jusqu'à ce que l'on constate qu'il était plus efficace de les implémenter en logiciel et de consacrer le silicium du processeur à des tâches plus fréquentes. Avec l'arrivée de co processeurs reconfigurables de grande capacité, cette situation doit être reévaluée : ces coprocesseurs ont désormais une capacité suffisante pour accélerer de manière importante le calcul d'une fonction élémentaire en virgule flottante double précision, sans pour autant devoir sacrifier pour cela des ressources de manière permanente. Cet article étudie le reciblage vers cette technologie d'opérateurs optimisés pour l'exponentielle et le logarithme jusqu'à la double précision.

Mots-clés: Fonctions élémentaires en virgule flottante, opérateur matériel, FPGA, exponentielle, logarithme. 


\section{Introduction}

Virtually all the computing systems that support some form of floating-point (FP) also include a floatingpoint mathematical library (libm) providing elementary functions such as exponential, logarithm, trigonometric and hyperbolic functions, etc. Modern systems usually comply with the IEEE-754 standard for floating-point arithmetic and offer hardware for basic arithmetic operations in single- and double-precision formats (32 bits and 64 bits respectively).

The question wether elementary functions should be implemented in hardware was controversial in the beginning of the PC era [15]. The literature indeed offers many articles describing hardware implementations of FP elementary functions $[8,21,10,2,18,19,20]$. In the early 80 s, Intel chose to include elementary functions to their first math co-processor, the 8087 .

However, for cost reasons, in this co-processor, as well as in its successors by Intel, Cyrix or AMD, these functions didnot use the hardware algorithm mentioned above, but were microcoded: In effect, the code for a libm was stored in the processor, with performance comparable to a software libm (one or two orders of magnitude slower than the basic operators). Soon, new algorithms, benefiting from technology advances, allowed to write software libm which were more accurate and faster than the hardware version. For instance, as memory went larger and cheaper, one could speed-up the computation using large tables (several kilobytes) of precomputed values. It would not be economical to cast such tables to silicon in a processor: The average computation will benefit much more from the corresponding silicon if it is dedicated to more cache, or more floating-point units for example. Besides, the hardware functions lacked the flexibility of the software ones, which could be optimized in context by advanced compilers.

These observations contributed to the move from CISC to RISC (Complex to Reduced Instruction Sets Computers) in the 90s. Intel themselves now also develop software libms for their processors that include a hardware libm [1]. Research on hardware elementary functions has since then mostly focused on approximation methods for fixed-point evaluation of functions $[11,16,12,6]$.

Lately, a new kind of programmable circuit has also been gaining momentum: The FPGA, for fieldprogrammable gate array. Designed to emulate arbitrary logic circuits, an FPGA consists of a very large number of configurable elementary blocks, linked by a configurable network of wires. A circuit emulated on an FPGA is typically one order of magnitude slower than the same circuit implemented directly in silicon, but FPGAs are reconfigurable and therefore offer a flexibility comparable to that of the microprocessor.

FPGAs have been used as co-processors to accelerate specific tasks, typically those for which the hardware available in processors is poorly suited. This, of course, is not the case of floating-point computing: an FP operation is, as already mentioned, typically ten times slower in FPGA than if computed in the highly optimized FPU of the processor. However, FPGA capacity has increased steadily with the progress of VLSI integration, and it is now possible to pack many FP operators on one chip: massive parallelism allows to recover the performance overhead [17], and accelerated FP computing has been reported in single precision [13], then in double-precision [3, 7]. Mainstream computer vendors such as Silicon Graphics and Cray build computers with FPGA accelerators - although to be honest, they do not advertise them (yet) as FP accelerators.

With this new technological target, the subject of hardware implementation of floating-point elementary functions becomes a hot topic again. Indeed, the literature reports that a single instance of an exponential [5] or logarithm [4] operator can provide ten times the performance of the processor, while consuming a small fraction of the resources of current FPGAs. The reason is that such an operator may perform most of the computation in optimized fixed point with specifically crafted datapaths, and is highly pipelined. However, the approach taken in $[4,5]$ uses a generic table-based approach [6], which doesn't scale well beyond single precision (its size grows exponentially).

In this article, we demonstrate a more algorithmic approach, which is a synthesis of much older works, including the Cordic/BKM family of algorithms [14], the radix-16 multiplicative normalization of [8], Chen's algorithm [21], an ad-hoc algorithm by Wong and Goto [19], and probably many others. All these approaches boil down to the same basic properties of the logarithm and exponential functions, and are synthesized in Section 2. The specificity of the FPGA hardware target are summarized in Section 3, and the optimized algorithms are detailed and evaluated in Section 4 (logarithm) and Section 5 (exponential). 


\section{$2 \quad$ Iterative exp and log}

Wether we want to compute the logarithm or the exponential, the idea common to most previous methods may be summarized by the following iteration. Let $\left(x_{i}\right)$ and $\left(l_{i}\right)$ be two given sequences of reals such that $\forall i, e^{l_{i}}=x_{i}$. It is possible to define two new sequences $\left(x_{i}^{\prime}\right)$ and $\left(l_{i}^{\prime}\right)$ as follows: $l_{0}^{\prime}$ and $x_{0}^{\prime}$ are such that $x_{0}^{\prime}=e^{l_{0}^{\prime}}$, and

$$
\forall i>0\left\{\begin{aligned}
l_{i+1}^{\prime} & =l_{i}+l_{i}^{\prime} \\
x_{i+1}^{\prime} & =x_{i} \times x_{i}^{\prime}
\end{aligned}\right.
$$
$\left.e^{l_{i+1}^{\prime}}\right)$

This iteration maintains the invariant $x_{i}^{\prime}=e^{l_{i}^{\prime}}$, since $x_{0}^{\prime}=e^{l_{0}^{\prime}}$ and $x_{i+1}=x_{i} x_{i}^{\prime}=e^{l_{i}} e^{l_{i}^{\prime}}=e^{l_{i}+l_{i}^{\prime}}=$

Therefore, if $x$ is given and one wants to compute $l=\log (x)$, one may define $l_{0}^{\prime}=x$, then read from a table a sequence $\left(l_{i}, x_{i}\right)$ such that the corresponding sequence $\left(l_{i}^{\prime}, x_{i}^{\prime}\right)$ converges to $(0,1)$. The iteration on $x_{i}^{\prime}$ is computed for increasing $i$, until for some $n$ we have $x_{n}^{\prime}$ sufficiently close to 1 so that one may compute its logarithm using the Taylor series $l_{i}^{\prime} \approx x_{n}^{\prime}-1-\left(x_{n}^{\prime}-1\right)^{2} / 2$, or even $l_{i}^{\prime} \approx x_{n}^{\prime}-1$. This allows to compute $\log (x)=l=l_{0}^{\prime}$ by the recurrence $(1)$ on $l_{i}^{\prime}$ for $i$ decreasing from $n$ to 0 .

Now if $l$ is given and one wants to compute its exponential, one will start with $\left(l_{0}^{\prime}, x_{0}^{\prime}\right)=(0,1)$. The tabulated sequence $\left(l_{i}, x_{i}\right)$ is now chosen such that the corresponding sequence $\left(l_{i}^{\prime}, x_{i}^{\prime}\right)$ converges to $\left(l, x=e^{l}\right)$.

There are also variants where $x_{i}^{\prime}$ converges from $x$ to 1 , meaning that (1) computes the reciprocal of $x$ as the product of the $x_{i}$. Several of the aforementioned papers explicitely propose to use the same hardware to compute the reciprocal $[8,19,14]$.

The various methods presented in the literature vary in the way they unroll this iteration, in what they store in tables, and in how they chose the value of $x_{i}$ to minimize the cost of multiplications. Comparatively, the additions in the $l_{i}^{\prime}$ iteration are less expensive.

Let us now study the optimization of such an iteration for an FPGA platform.

\section{A primer on arithmetic for FPGAs}

We assume the reader has basic notions about the hardware complexity of arithmetic blocks such as adders, multipliers, and tables in VLSI technology (otherwise see textbooks like [9]), and we highlight here the main differences when implementing a hardware algorithm on an FPGA.

- An FPGA consists of tens of thousand of elementary blocks, laid out as a square grid. This grid also includes routing channels which may be configured to connect blocks together almost arbitrarily.

- The basic universal logic element in most current FPGAs is the 4-input Look-Up Table (LUT), a small 16-bit memory whose content may be set at configuration time. Thus, any 4-input boolean function can be implemented by filling a LUT with the appropriate value. More complex functions can be built by wiring LUTs together.

For our purpose, as we will use tables of precomputed values, it means that 4 -input, $n$-output tables make the optimal use of the basic structure of the FPGA. A table with 5 inputs is twice as large as a table with 4 inputs, and a table with 3 inputs is not smaller.

- As addition is an ubiquitous operation, the elementary blocks contain additional circuitry dedicated to carry propagation between neighbouring LUTs. This allows to implement an $n$-bit adder in $n$ LUTs only. Besides, carry propagation this way is much faster than if it would use the routing channels. A consequence is that there is no need for carry-save representation of intermediate results: the plain carry-propagate adder is smaller, and faster for all but very large additions.

- In the elementary block, each LUT is followed by a 1-bit register, which may be used or not. For our purpose it means that turning a combinatorial circuit into a pipelined one means using a resource that is present, not using more resources (in practice, however, a pipelined circuit will consume marginally more resources).

- Recent FPGAs include a limited number of small multipliers or mult-accumulators, typically for 16 bits times 16 bits. In this work, we choose not to use them. 


\section{A hardware logarithm operator}

\subsection{First range reduction and reconstruction}

The logarithm is only defined for positive floating-point numbers, and does not overflow nor underflow. Exceptional cases are therefore trivial to handle and will not be mentioned further. A positive input $X$ is written in floating-point format $X=2^{E_{X}-E_{0}} \times 1 . F_{X}$, where $E_{X}$ is the stored exponent, $F_{X}$ is the stored significand, and $E_{0}$ is the exponent bias (as per the IEEE-754 standard).

Now we obviously have $\log (X)=\log \left(1 . F_{X}\right)+\left(E_{X}-E_{0}\right) \cdot \log 2$. However, if we use this formula, the logarithm of $1-\epsilon$ will be computed as $\log (2-2 \epsilon)-\log (2)$, meaning a catastrophic cancellation. To avoid this case, the following error-free transformation is applied to the input:

$$
\left\{\begin{array}{lll}
Y_{0}=1 . F_{X}, & E=E_{X}-E_{0} & \text { when 1. } F_{X} \in[1,1.5), \\
Y_{0}=\frac{1 . F_{X}}{2}, & E=E_{X}-E_{0}+1 & \text { when 1. } F_{X} \in[1.5,2) .
\end{array}\right.
$$

And the logarithm is evaluated as follows:

$$
\log (X)=\log \left(Y_{0}\right)+E \cdot \log 2 \quad \text { with } \quad Y_{0} \in[0.75,1.5)
$$

Then $\log \left(Y_{0}\right)$ will be in the interval $(-0.288,0.406)$. This interval is not very well centered around 0 , and other authors use in (2) a value closer to $\sqrt{2}$, as a well-centered interval allows for a better approximation by a polynomial. We prefer that the comparison resumes to testing the first bit of $F$, called FirstBit in the following (see Figure 1).

Now consider equation (3), and let us discuss the normalization of the result: we need to know which will be the exponent of $\log (X)$. There are two mutually exclusive cases.

- Either $E \neq 0$, and there will be no catastrophic cancellation in (3). We may compute $E \log 2$ as a fixed-point value of size $w_{F}+w_{E}+g$ (where $g$ is a number of guard bit to be determined). This fixed-point sum will be added to a fixed-point value of $\log \left(Y_{0}\right)$ on $w_{F}+1+g$ bits, then a combined leading-zero-counter and barrel-shifter will determine the exponent and mantissa of the result. In this case the shift will be at most of $w_{E}$ bits.

- Or, $E=0$. In this case the logarithm of $Y_{0}$ may vanish, which means that a shift to the left will be needed to normalize the result ${ }^{1}$.

- If $Y_{0}$ is close enough to 1 , specifically if $Y_{0}=1+Z_{0}$ with $\left|Z_{0}\right|>w_{F} / 2$, the left shift may be predicted thanks to the Taylor series $\log (1+Z) \approx Z-Z^{2} / 2$ : its value is the number of leading zeroes (if FirstBit=0) or leading ones (if FirstBit=1) of $Y_{0}$. We actually perform the shift before computing the Taylor series, to maximize the accuracy of this computation. This actually consists of two shifts, one on $Z$ and one on $Z^{2}$, as seen on Figure 1 .

- Or, $E=0$ but $Y_{0}$ is not sufficiently close to 1 and we have to use a range reduction, knowing that it will cancel at most $w_{F} / 2$ significant bits. The simpler is to use the same LZC/barrel shifter than in the first case, which now has to shift by $w E+w_{F} / 2$.

Figure 1 depicts the corresponding architecture. A detailed error analysis will be given in 4.3.

\subsection{Multiplicative range reduction}

This section describes the work performed by the box labelled Range Reduction on Figure 1. Consider the centered mantissa $Y_{0}$. If FirstBit $=0, Y_{0}$ has the form $1.0 \mathrm{xx} \ldots \mathrm{xx}$, and its logarithm will eventually be positive. If FirstBit $=1, Y_{0}$ has the form $0.11 \mathrm{xx} \ldots \mathrm{xx}$ (where the first 1 is the former implicit 1 of the floating-point format), and its logarithm will be negative.

Let $A_{0}$ be the first 5 bits of the mantissa (including FirstBit). $A_{0}$ is used to index a table which gives an approximation $\widetilde{Y_{0}^{-1}}$ of the inverse of $Y_{0}$ on 6 bits. Noting $\widetilde{Y_{0}}$ the mantissa where the bits lower

\footnotetext{
${ }^{1}$ This may seem a lot of shifts to the reader. Consider that there are barrel shifters in all the floating-point adders: In a software logarithm, you have many more hidden shifts, and you pay for them even when you don't use them.
} 


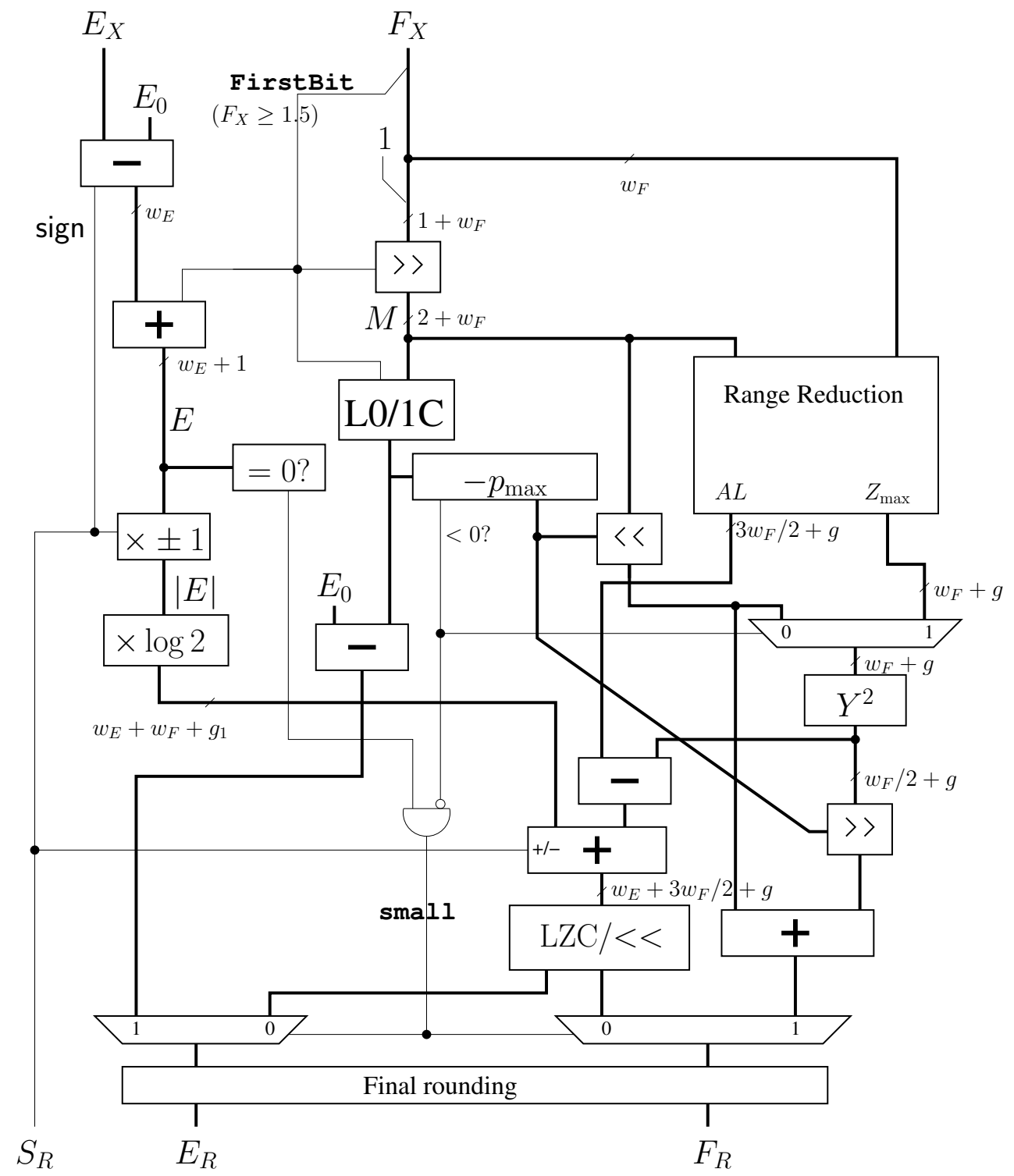

Figure 1: Overview of the logarithm

than those of $A_{0}$ are zeroed $\left(\widetilde{Y_{0}}=1.0\right.$ aaaaa or $\widetilde{Y_{0}}=0.11$ aaaaa, depending on FirstBit $)$, the first inverse table stores

$$
\widetilde{Y_{0}^{-1}}=2^{-5}\left\lceil\frac{2^{6}}{\widetilde{Y_{0}}}\right\rceil
$$

The reader may check that these values ensure $Y_{0} \times \widetilde{Y_{0}^{-1}} \in\left[1,1+2^{-4}\right]$. Therefore we define $Y_{1}=$ $1+Z_{1}=Y_{0} \times \widetilde{Y_{0}^{-1}}$ and $0 \leq Z_{1}<2^{-p_{1}}$, with $p_{1}=4$. The multiplication $Y_{0} \times \widetilde{Y_{0}^{-1}}$ is a rectangular one, since $\widetilde{Y_{0}^{-1}}$ is a 6 -bit only number. $A_{0}$ is also used to index a first logarithm table, that contains an accurate approximation $L_{0}$ of $\log \left(\widetilde{Y_{0}^{-1}}\right)$ (the exact precision will be given later). This provides the first step of an iteration similar to (1):

$$
\log \left(Y_{0}\right)=\log \left(Y_{0} \times \widetilde{Y_{0}^{-1}}\right)-\log \left(\widetilde{Y_{0}^{-1}}\right)=\log \left(1+Z_{1}\right)-\log \left(\widetilde{Y_{0}^{-1}}\right)=\log \left(Y_{1}\right)-L_{0}
$$

and the problem is reduced to evaluating $\log \left(Y_{1}\right)$. 
The following iterations will similarly build a sequence $Y_{i}=1+Z_{i}$ with $0 \leq Z_{i}<2^{-p_{i}}$. Note that the sign of $\log \left(Y_{0}\right)$ will always be given by that of $L_{0}$, which is itself entirely defined by FirstBit. However, $\log \left(1+Z_{1}\right)$ will be non-negative, as will be all the following $Z_{i}$ (see Figures 2 and 3 ).

Let us now define the general iteration, starting from $i=1$. Let $A_{i}$ be the subword composed of the $\alpha_{i}$ leading bits of $Z_{i}$ (bits of absolute weight $2^{-p_{i}-1}$ to $2^{-p_{i}-\alpha_{i}}$ ). $A_{i}$ will be used to address the logarithm table $L_{i}$. As suggested in Section 3, we choose $\alpha_{i}=4 \forall i>0$ to minimize resource usage, but another choice could lead to a different area/speed tradeoff. For instance, the architecture by Wong and Goto [19] takes $\alpha_{i}=10$. Note that we used $\alpha_{0}=5$, because $\alpha_{0}=4$ would lead to $p_{1}=2$, which seems a worse tradeoff.

The following iterations no longer use an inverse table: An approximation of the inverse of $Y_{i}=1+Z_{i}$ is defined by

$$
\widetilde{Y_{i}^{-1}}=1-A_{i}+\epsilon_{i}
$$

The term $\epsilon_{i}$ is a single bit that will ensure that $\widetilde{Y_{i}^{-1}} \times Y_{i} \geq 1$. We define it as

$$
\begin{cases}\text { when } \alpha_{i}+1<p_{i} & \left.\epsilon_{i}=2^{-p_{i}-\alpha_{i}-1} \quad \text { (one half-ulp of } A_{i}\right) \\ \text { if } \alpha_{i}+1 \geq p_{i} & \begin{cases}\text { if } M S B\left(A_{i}\right)=0 \quad \epsilon_{i}=2^{-p_{i}-\alpha_{i}-1} \quad \text { (one half-ulp of } A_{i} \text { ) } \\ \text { if } M S B\left(A_{i}\right)=1 \quad \epsilon_{i}=2^{-p_{i}-\alpha_{i}} \quad \text { (one ulp of } A_{i} \text { ) }\end{cases} \end{cases}
$$

This definition seems contrived, but is easy to implement in hardware. Besides, the case $\alpha_{i}+1 \geq p_{i}$ happens only once in practice. From (5) and (6), it is possible to show that the following holds:

Lemma 4.1

$$
0 \leq Y_{i+1}=1+Z_{i+1}=\widetilde{Y_{i}^{-1}} \times Y_{i}<1+2^{-p_{i}-\alpha_{i}+1}
$$

The proof is not difficult, considering (8) below, but is too long to be exposed here.

In other words, we ensure $p_{i+1}=p_{i}+\alpha_{i}-1$. Or, using $\alpha_{i}$ bits of table address, we are able to zero out $\alpha_{i}-1$ bits of our argument. This is slightly better than [19] where $\alpha_{i}-2$ bits are zeroed. Approaches inspired by division algorithms [8] are able to zero $\alpha_{i}$ bits (one radix- $2^{\alpha_{i}}$ digit), but at a higher hardware cost due to the need for signed digit arithmetic.

With $\alpha_{i}=4$ on an FPGA, the main cost is not in the $L_{i}$ table (at most one LUT per table output bit), but in the multiplication. However, a full multiplication is not needed. Noting $Z_{i}=A_{i}+B_{i}\left(B_{i}\right.$ consists of the lower bits of $\left.Z_{i}\right)$, we have $1+Z_{i+1}=\widetilde{Y_{i}^{-1}} \times\left(1+Z_{i}\right)=\left(1-A_{i}+\epsilon_{i}\right) \times\left(1+A_{i}+B_{i}\right)$, hence

$$
Z_{i+1}=B_{i}-A_{i} Z_{i}+\epsilon_{i}\left(1+Z_{i}\right)
$$

Here the multiplication by $\epsilon_{i}$ is just a shift, and the only real multiplication is the product $A_{i} Z_{i}$ : The full computation of (8) amounts to the equivalent of a rectangular multiplication of $\left(\alpha_{i}+2\right) \times s_{i}$ bits. Here $s_{i}$ is the size of $Z_{i}$, which will vary between $w_{F}$ and $3 w_{F} / 2$ (see below).

An important remark is that Lemma 4.1 still holds if the product is truncated. Indeed, in the architecture, we will need to truncate it to limit the size of the computation datapath. Let us now address this question.

We will stop the iteration as soon as $Z_{i}$ is small enough for a second-order Taylor formula to provide sufficient accuracy (this also defines the threshold on leading zeroes/ones at which we choose to use the path computing $Z_{0}-Z_{0}^{2} / 2$ directly). In $\log \left(1+Z_{i}\right) \approx Z_{i}-Z_{i}^{2} / 2+Z_{i}^{3} / 3$, with $Z_{i}<2^{-p_{i}}$, the third-order term is smaller than $2^{-3 p_{i}-1}$. We therefore stop the iteration at $p_{\max }$ such that $p_{\max } \geq\left\lceil\frac{w_{F}}{2}\right\rceil$. This sets the target absolute precision of the whole datapath to $p_{\max }+w_{F}+g \approx\left\lceil 3 w_{F} / 2\right\rceil+g$. The computation defined by (8) increases the size of $Z_{i}$, which will be truncated as soon as its LSB becomes smaller than this target precision. Figures 2 and 3 give an instance of this datapath in single and double precision respectively. Note that the architecture counts as many rectangular multipliers as there are stages, and may therefore be fully pipelined. Reusing one single multiplier would be possible, and would save a significant amount of hardware, but a high-throughput architecture is preferable.

Finally, at each iteration, $A_{i}$ is also used to index a logarithm table $L_{i}$ (see Figures 2 and 3 ). All these logarithms have to be added, which can be done in parallel to the reduction of $1+Z_{i}$. However, the adders are smaller if the addition is performed in the order of decreasing $i$, in parallel with the computation of $Z_{\max }^{2}$. The output of the Range Reduction box is the sum of $Z_{\max }$ and this sum of tabulated logarithms, so it only remains to subtract the second-order term (Figure 1). 


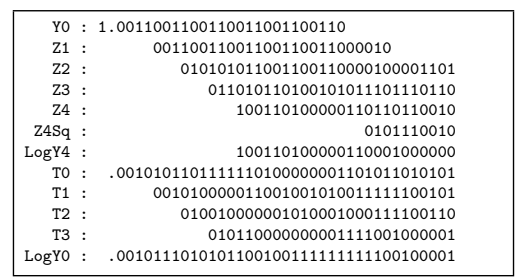

Figure 2: Single-precision computation of $\log \left(Y_{0}\right)$ for $Y_{0}=1.2$

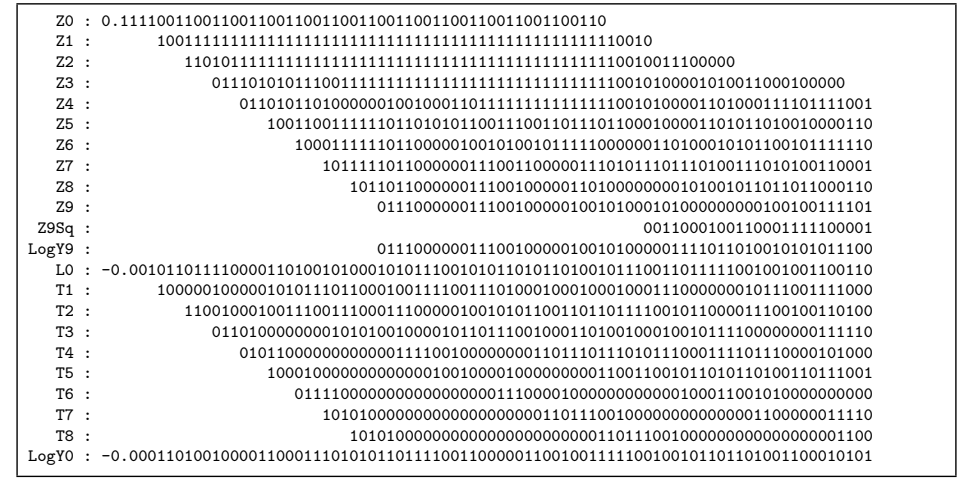

Figure 3: Double-precision computation of $\log \left(Y_{0}\right)$ for $Y_{0}=0.95$.

\subsection{Error analysis}

We compute $E \log 2$ with $w_{E}+w_{F}+g 1$ precision, and the sum $E \log 2+\log Y_{0}$ cancels at most one bit, so $g_{1}=2$ ensures faithful accuracy of the sum, assuming faithful accuracy of $\log Y_{0}$.

In general, the computation of $\log Y_{0}$ is much too accurate: as illustrated by Figure 2, the most significant bit of the result is that of the first non-zero $L_{i}\left(L_{0}\right.$ in the example), and we have computed almost $w_{F} / 2$ bits of extra accuracy. The errors due to the rounding of the $L_{i}$ and the truncation of the intermediate computations are absorbed by this extra accuracy. However, two specific worst-case situation require more attention.

- When $Z_{0}<2^{-p_{\max }}$, we compute $\log Y_{0}$ directly as $Z_{0}-Z_{0}^{2} / 2$, and this is the sole source of error. The shift that brings the leading one of $\left|Z_{0}\right|$ in position $p_{\max }$ ensures that this computation is done on $w_{F}+g$ bits, hence faithful rounding.

- The real worst case is when $Y_{0}=1-2^{-p_{\max }+1}$ : in this case we use the range reduction, knowing that it will cancel $p_{\max }-1$ bits of $L_{0}$ one one side, and accumulate rounding errors on the other side. Since we have max stages, each contributing at most 1.5 ulp of error, we need $g=\left\lceil\log _{2}\left(1.5 p_{\max }\right)\right\rceil$ guard bits. For double-precision, this gives $g=4$.

\subsection{Remarks on the $L_{i}$ tables}

When one looks at the $L_{i}$ tables, one notices that some of their bits are constantly zeroes: indeed they hold $L_{i} \approx \log \left(1-\left(A_{i}-\epsilon_{i}\right)\right)$ wich can for larger $i$ be approximated by a Taylor series. We chose to leave the task of optimizing out these zeroes to the logic synthesizer. A natural idea would also be to store only $\log \left(1-\left(A_{i}-\epsilon_{i}\right)\right)+\left(A_{i}-\epsilon_{i}\right)$, and construct $L_{i}$ out of this value by subtracting $\left(A_{i}-\epsilon_{i}\right)$. However, the delay and LUT usage of this reconstruction would in fact be higher than that of storing the corresponding bits. As a conclusion, with the FPGA target, the simpler approach is also the better. The same remark will apply to the tables of the exponential operator. 


\section{A hardware exponential algorithm}

\subsection{Initial range reduction and reconstruction}

The range reduction for the exponential operator is directly inspired from the method presented in [5]. The first step transforms the floating-point input $X$ into a fixed-point number $X_{\text {fix }}$ thanks to a barrel shifter. Indeed, if $E_{X}>w_{E}-1+\log _{2}(\log 2)$, then the result overflows, while if $E_{X}<0$, then $X$ is close to zero and its exponential will be close to $1+X$, so we can lose the bits of $X$ of absolute weight smaller than $2^{-w_{F}-g}, g$ being the number of guard bits required for the operator (typically 3 to 5 bits). Thus $X_{\text {fix }}$ will be a $w_{E}+w_{F}+g$-bit number, obtained by shifting the mantissa $1 . F_{X}$ by at most $w_{E}-1$ bits on the left and $w_{F}+g$ bits on the right.

This fixed-point number is then reduced in order to obtain an integer $E$ and a fixed-point number $Y$ such that $X \approx E \cdot \log 2+Y$ and $0 \leq Y<1$. This is achieved by first multiplying the most significant bits of $X_{\mathrm{fix}}$ by $1 / \log 2$ and then truncating the result to obtain $E$. Then $Y$ is computed as $X_{\mathrm{fix}}-E \cdot \log 2$, requiring a rectangular multiplier.

After computing $e^{Y}$ thanks to the iterative algorithm detailed in the next section, we have $e^{X} \approx$ $e^{X_{\mathrm{fix}}}=e^{Y} \cdot 2^{E}$, therefore a simple renormalization and rounding step may reconstruct the final result.

The left half of Figure 4 presents the overall architecture of the exponential operator. Details for the computation of $e^{Y}-1$ are given in the other half of the figure and presented in the next section.

\subsection{Iterative algorithm for the fixed-point exponential}

Starting with the fixed-point operand $Y \in[0,1)$, we want to compute $e^{Y}-1$. Define $Y_{0}=Y$. Each iteration starts with a fixed-point number $Y_{i}$, which (as for the logarithm operator) is then split into $A_{i}$ and $B_{i}$ of $\alpha_{i}$ and $\beta_{i}$ bits respectively.

We then want to compute an approximation of $e^{Y_{i}}$, which we note $\widetilde{e}^{Y_{i}}$ using only the subword $A_{i}$. To this effect, we address two tables by the $\alpha_{i}$ bits of $A_{i}$, the first one containing the values of $\widetilde{e^{Y_{i}}}-1$ rounded to only $\alpha_{i}$ bits, and the second one holding $L_{i}=\log \left(\widetilde{e^{Y_{i}}}\right)$ rounded to $\alpha_{i}+\beta_{i}$ bits.

We can check that $L_{i}$ is quite close to $Y_{i}$, and that computing $Y_{i+1}$ as the difference $Y_{i}-L_{i}$ will result in cancelling the $\alpha_{i}-1$ most significant bits of $Y_{i}$. The number $Y_{i+1}$ fed into the next iteration is therefore a $1+\beta_{i}$-bit number.

Iterations are performed up to $Y_{k}$, where $Y_{k}$ is small enough to evaluate $e^{Y_{k}}-1$ thanks to a simple table or a Taylor approximation.

The reconstruction is then quite straightforward: considering that we have obtained the result $e^{Y_{i+1}}-1$ from the previous iterations, we first compute the product of $\widetilde{e^{Y_{i}}}-1$, from the first table addressed by $A_{i}$, by $e^{Y_{i+1}}-1$. This is done by a rectangular multiplier, since $\widetilde{e^{Y_{i}}}-1$ is tabulated on $\alpha_{i}$ bits only. Finally adding the same $\widetilde{e^{Y_{i}}}-1$ and $e^{Y_{i+1}}-1$ to the product, we obtain:

$$
\begin{aligned}
\left(\widetilde{e^{Y_{i}}}-1\right) \times\left(e^{Y_{i+1}}-1\right)+\left(\widetilde{e^{Y_{i}}}-1\right)+\left(e^{Y_{i+1}}-1\right) & =\widetilde{e^{Y_{i}}} \cdot e^{Y_{i+1}}-1 \\
& =\widetilde{e^{Y_{i}}} \cdot e^{Y_{i}-L_{i}}-1 \\
& =\widetilde{e}^{Y_{i}} \cdot e^{Y_{i}} \cdot e^{-\log \left(\widetilde{e^{Y_{i}}}\right)}-1 \\
& =e^{Y_{i}}-1 .
\end{aligned}
$$

This way, the $k$ steps of reconstruction finally give the result $e^{Y_{0}}-1=e^{Y}-1$. The detailed architecture of this iterative method is presented Figure 4.

We have performed a detailed error analysis of this algorithm to ensure the faithful rounding of the final result. Due to space restrictions, and considering its similarity with the one for the logarithm, this analysis is not presented in this article.

\section{Area and performance}

The presented algorithms are implemented as $\mathrm{C}++$ programs that input $w_{E}, w_{F}$ and possibly the $\alpha_{i}$, compute the various parameters of the architecture, and output synthesisable VHDL. Some values of 


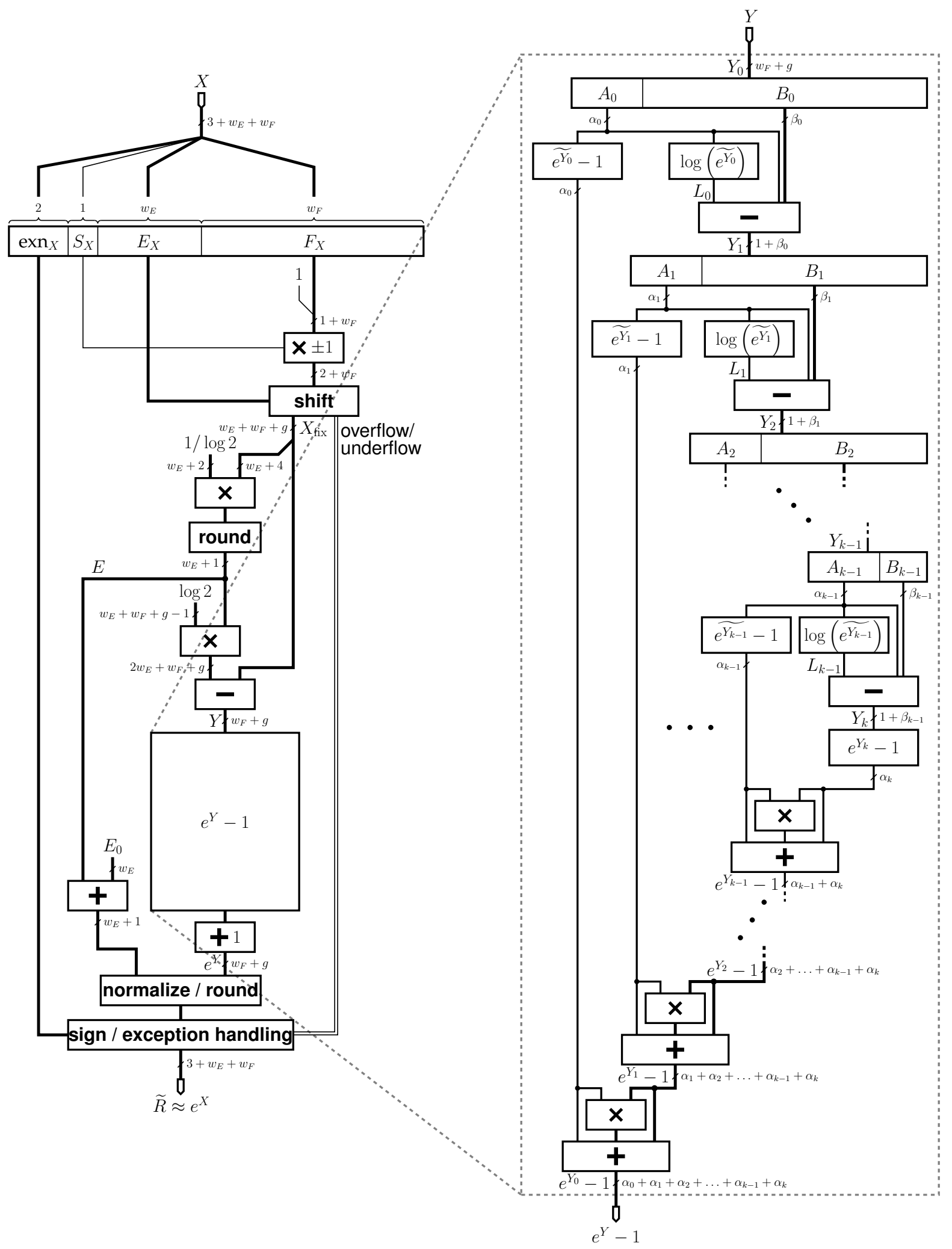

Figure 4: Overview of the exponential 
area and delay (obtained using Xilinx ISE/XST 8.2 for a Virtex-II XC2V1000-4 FPGA) are given in Table 1 (where a slice is a unit containing two LUTs).

As expected, the operators presented here are smaller but slower than the previously reported ones. More importantly, their size is more or less quadratic with the precision, instead of exponential for the previously reported ones. This allows them to scale up to double-precision. For comparison, the FPGA used as a co-processor in the Cray XD1 system contains more than 23,616 slices, and the current largest available more than 40,000, so the proposed operators consume about one tenth of this capacity. To provide another comparison, our operators consume less than twice the area of an FP multiplier for the same precision reported in [17].

\begin{tabular}{|c||c|c||c|c|}
\hline \multicolumn{5}{|c|}{ Exponential } \\
\hline \multirow{2}{*}{$\begin{array}{c}\text { Format } \\
\left(w_{E}, w_{F}\right)\end{array}$} & This work & Previous $[5]$ \\
\cline { 2 - 5 } & Area & Delay & Area & Delay \\
\hline \hline$(7,16)$ & $\mathbf{4 7 2}$ & $\mathbf{1 1 8}$ & 480 & 69 \\
$(8,23)$ & $\mathbf{7 2 8}$ & $\mathbf{1 2 3}$ & 948 & 85 \\
$(9,38)$ & $\mathbf{1 2 4 2}$ & $\mathbf{1 7 5}$ & - & - \\
$(11,52)$ & $\mathbf{2 0 4 5}$ & $\mathbf{2 2 9}$ & - & - \\
\hline \hline \multicolumn{5}{|c|}{ Logarithm } \\
\hline Format & \multicolumn{3}{|c|}{ This work } & Previous $[4]$ \\
\cline { 2 - 5 }$\left(w_{E}, w_{F}\right)$ & Area & Delay & Area & Delay \\
\hline \hline$(7,16)$ & $\mathbf{4 8 5}$ & $\mathbf{8 2}$ & 627 & 56 \\
$(8,23)$ & $\mathbf{8 2 0}$ & $\mathbf{1 0 0}$ & 1368 & 69 \\
$(9,38)$ & $\mathbf{1 9 6 0}$ & $\mathbf{1 5 9}$ & - & - \\
$(11,52)$ & $\mathbf{3 1 2 2}$ & $\mathbf{2 0 1}$ & - & - \\
\hline
\end{tabular}

Table 1: Area (in Virtex-II slices) and delay (in ns) of implementation on a Virtex-II 1000

These operators will be easy to pipeline to function at the typical frequency of FPGAs $-100 \mathrm{MHz}$ for the middle-range FPGAs targeted here, $150 \mathrm{MHz}$ for the best current ones. The pipeline depth is expected to be quite long, up to about 30 cycles for double precision. Such lengths are typical of FPGA floating-point applications (a double-precision multiplier is reported at 20 to 39 cycles in [17]) - and are less than the number of cycles it takes in software. As mentioned in [5] and [4], one exponential or logarithm per cycle at $100 \mathrm{MHz}$ is ten times the throughput of a $3 \mathrm{GHz}$ Pentium.

\section{Conclusion and future work}

By retargeting an old family of algorithms to the specific fine-grained structure of FPGAs, this work shows that elementary functions up to double precision can be implemented in a small fraction of current FPGAs. The resulting operators have low resource usage and high troughput, but long latency. The long latency is not really a problem for the envisioned applications. When used as co-processors, FPGAs are limited by their input/output bandwidth to the processor, and bringing elementary functions on-board will help conserve this bandwidth.

The same principles can be used to compute sine and cosine and their inverses, using the complex identity $e^{j x}=\cos x+j \sin x$. Its architectural translation, of course, is not trivial. Besides the main cost with trigonometric functions is actually in the argument reduction involving the transcendental number $\pi$. It probably makes more sense to implement functions such as $\sin (\pi x)$ and $\cos (\pi x)$. A detailed study of this issue remains to be done.

\section{References}

[1] C. S. Anderson, S. Story, and N. Astafiev. Accurate math functions on the intel IA-32 architecture: A performance-driven design. In 7th Conference on Real Numbers and Computers, pages 93-105, 2006. 
[2] M. Cosnard, A. Guyot, B. Hochet, J. M. Muller, H. Ouaouicha, P. Paul, and E. Zysmann. The FELIN arithmetic coprocessor chip. In Eighth IEEE Symposium on Computer Arithmetic, pages 107-112, 1987.

[3] M. deLorimier and A. DeHon. Floating-point sparse matrix-vector multiply for FPGAs. In ACM/SIGDA Field-Programmable Gate Arrays, pages 75-85. ACM Press, 2005.

[4] J. Detrey and F. de Dinechin. A parameterizable floating-point logarithm operator for FPGAs. In 39th Asilomar Conference on Signals, Systems 83 Computers. IEEE Signal Processing Society, November 2005.

[5] J. Detrey and F. de Dinechin. A parameterized floating-point exponential function for FPGAs. In IEEE International Conference on Field-Programmable Technology (FPT'05). IEEE Computer Society Press, December 2005.

[6] J. Detrey and F. de Dinechin. Table-based polynomials for fast hardware function evaluation. In 16th Intl Conference on Application-specific Systems, Architectures and Processors. IEEE Computer Society Press, July 2005.

[7] Y. Dou, S. Vassiliadis, G. K. Kuzmanov, and G. N. Gaydadjiev. 64-bit floating-point FPGA matrix multiplication. In ACM/SIGDA Field-Programmable Gate Arrays. ACM Press, 2005.

[8] M. Ercegovac. Radix-16 evaluation of certain elementary functions. IEEE Transactions on Computers, C-22(6):561-566, June 1975.

[9] M. D. Ercegovac and T. Lang. Digital Arithmetic. Morgan Kaufmann, 2003.

[10] P.M. Farmwald. High-bandwidth evaluation of elementary functions. In Fifth IEEE Symposium on Computer Arithmetic, pages 139-142, 1981.

[11] H. Hassler and N. Takagi. Function evaluation by table look-up and addition. In 12th IEEE Symposium on Computer Arithmetic, pages 10-16, Bath, UK, 1995. IEEE.

[12] D.U. Lee, A.A. Gaffar, O. Mencer, and W. Luk. Optimizing hardware function evaluation. IEEE Transactions on Computers, 54(12):1520-1531, December 2005.

[13] G. Lienhart, A. Kugel, and R. Männer. Using floating-point arithmetic on FPGAs to accelerate scientific N-body simulations. In FPGAs for Custom Computing Machines. IEEE, 2002.

[14] J.-M. Muller. Elementary Functions, Algorithms and Implementation. Birkhauser, Boston, $1997 / 2005$.

[15] G. Paul and M. W. Wilson. Should the elementary functions be incorporated into computer instruction sets? ACM Transactions on Mathematical Software, 2(2):132-142, June 1976.

[16] J.E. Stine and M.J. Schulte. The symmetric table addition method for accurate function approximation. Journal of VLSI Signal Processing, 21(2):167-177, 1999.

[17] K. Underwood. FPGAs vs. CPUs: Trends in peak floating-point performance. In ACM/SIGDA Field-Programmable Gate Arrays. ACM Press, 2004.

[18] W. F. Wong and E. Goto. Fast evaluation of the elementary functions in double precision. In Twenty-Seventh Annual Hawaii International Conference on System Sciences, pages 349-358, 1994.

[19] W. F. Wong and E. Goto. Fast hardware-based algorithms for elementary function computations using rectangular multipliers. IEEE Transactions on Computers, 43(3):278-294, March 1994.

$[20]$ W.F. Wong and E. Goto. Fast evaluation of the elementary functions in single precision. IEEE Transactions on Computers, 44(3):453-457, March 1995.

[21] C. Wrathall and T. C. Chen. Convergence guarantee and improvements for a hardware exponential and logarith evaluation scheme. In Fourth IEEE Symposium on Computer Arithmetic, pages 175$182,1978$. 